\title{
HIPHOP IM CANDY SHOP. ÜBERLEGUNGEN ZUR POPULÄREN STIMME
}

\author{
Christian Bielefeldt
}

»Voices, not songs, hold the key to our pop pleasures« (Frith 1996: 201).

»Vocal performance has been evaluated largely as a means of expressing an sinner self. [...] she gave it all!<, we say, implying that she gave from herself, from what might be behind the sartistic « curtain« (Danielsen 1997: 279).

Der Videoclip zu 50 Cents Song "Candy Shop« ist ein einziger süßer Traum. Und ein Traum von Süße; eine Wunschphantasie voller kandierter Früchte und wie heiße, dunkle Sirupströme dahinschmelzender junger Frauen, präsentiert in einer Ästhetik, in der Zucker gleich Sex ist und Sex ein reines Zuckerschlecken (ein nächtlicher Traum dabei, der in dunklen Gemäuern handelt und mit einem Schluss aufwartet, über den noch zu sprechen ist). In diesem Traum erklingt, sich abhebend vom lockenden Refrain der G-UnitLady Olivia, die Stimme des Rappers Curtis " 50 Cent « Jackson.

Wenn ich 50 Cent rappen höre, vernehme ich auf einem Ohr eine rappende - und im schlichten Refrain singende - Stimme, die Töne und Worte produziert sowie musikalische und sprachliche Kodes aufrufende Zeichen. Auf diesem Ohr höre ich Stimme als ästhetische Konstruktion, als einen nach bestimmten Prinzipien geordneten, ausdruckshaften Zusammenhang von Sprache und Musik. Auf dem anderen Ohr höre ich Stimme als Sound, als klingenden Stimm-Laut mit spezifischer Farbe und Charakteristik. Über diese stimmliche Dimension lässt sich, weil Sound schwer sprachlich zu qualifizieren und noch schwerer zu objektivieren ist, nur in Annäherungen sprechen. Ich höre bei 50 Cent eine eher jungenhafte als männliche Stimme, aber im tiefen Register mit vergleichsweise wenig Geräuschanteil für einen Rapper in der Tradition des Gangsta- und Hardcore-Rap. Ich registriere bei inm die Tendenz zum Undeutlichen, die sich in seiner stark verschliffenen Aussprache manifestiert, der Slang-Färbung der Vokale sowie in der nur annäherungsweise Tonhöhen markierenden Intonation und der rhythmischen Unschärfe der Rap-Verse. Und ich höre - und spüre dabei - eine schwere 
und zugleich geschmeidige, extrem entspannte, dabei niemals auftrumpfende Lässigkeit, wie aus der definitiven Nachzeit aller großen Aufregungen erwachsen, hinter der ich eine nur halb ausgespielte, für alle notwendigen Fälle in Reserve gehaltene Klangpotenz vermute. Unwillkürlich gleiche ich diese Eindrücke dann ab mit dem, was ich weiß über den Megaseller und Superstar 50 Cent, vom biographischen Hintergrund mit seinen dunkleren Flecken über den überragenden Erfolg der beiden Alben Get Rich Or Die Tryin' (2003) und Massacre (2005) bis hin zum ersten Hollywood-Film: Wie weit bestätigt die Stimme das Bild, das ich mir von ihm gemacht habe, wie weit widerlegt sie es?

Wenn ich 50 Cent rappen höre, höre ich also zweierlei: ein Bedeutung transportierendes Stimm-Medium einerseits und andererseits eine charakteristische Signatur, eine spezifische stimmliche Praxis der Klangerzeugung mit schwer in Worte zu fassenden Eigenschaften. Und diese zweite, komplexe Signatur nehme ich dabei als "Spur des Lebendig-Seins (Kolesch 2003: 267), als direkten, glaubwürdigen Hinweis auf den Charakter des singenden Subjekts. Ich höre und befrage die Stimme des Rappers als Medium von Persönlichkeit.

Die Stimme als Zugang zum Selbst des Sängers zu hören, gilt als gängige Praxis der Stimmrezeption in populärer Musik. Wie aber, in welcher der vielen Stimmen in der Stimme, wird dieses Selbst hörbar? Eben nicht, glauben wir den eingangs zitierten Worten von Anne Danielsen, auf der Ebene der für Ausdruck eigentlich zuständigen Medien Sprache (Songtext) oder musikalische Struktur (Song). Sondern in einer zusätzlichen, einer stimmlichen Dimension »hinter dem >künstlerischen< Vorhang «. Nicht die Stimme als klingende ästhetische Struktur, sondern eine zweite, andere Stimme, die wir als Klangsignatur eines authentischen »inneren Selbst « verstehen (wollen), wertet das Stimme-Hören auf zur intensiven Nah-Interaktion mit dem Selbst des Künstlers und, nach Simon Friths oben zitiertem Postulat, überhaupt zur primären Erfahrung und Motivation des Pophörens.

Wie aber entsteht dieser offenbar von vielen Hörern hergestellte Zusammenhang zwischen Stimmcharakteristik und Sängerpersönlichkeit; welche Hör- und Deutungsprozesse machen es wahrscheinlich, die zweite, personale Stimme als Authentizitätssignatur zu hören? Mehr oder weniger verdeckt taucht an diesem Punkt der Beschäftigung mit Pop-Stimme(n) in der Regel das Konzept des "Genogesangs« von Roland Barthes (1990: 302) auf, als eines undomestizierten Gesangs des Körpers und nicht der Kultur. Verlängert in den Kontext der populären Stimme, wäre demnach die physiologische Stimme, verstanden als klangliche Extension des empirischen, materiellen Körpers, die maßgebliche Instanz des Persönlichen. Im Folgenden 
wird dieser Auffassung widersprochen und ihr ein konkurrierendes Modell gegenübergestellt, ausgehend von der Annahme, dass gerade die personale Stimme immer auch als auditives Gedächtnisfeld zu hören ist, auf dem historische und aktuelle, individuelle und kollektive Gesten, Stile und Ausdrucksmodelle der Stimme miteinander konkurrieren, interagieren und sich gegenseitig kommentieren, und dies im Übrigen keineswegs nur in der populären Musik. Der hiermit angerissene Problembereich reicht weit über musikwissenschaftliche Zuständigkeiten hinaus. Ich will im Verlauf des vorliegenden Beitrags darum auf Bausteine eines Diskurses zurückgreifen, der bislang seine Schwerpunkte eher in den Kultur-, Literatur- und Theaterwissenschaften setzte. Seine Anschlussfähigkeit für die Popmusikforschung sei damit zugleich auf die Probe gestellt.

\section{Stimme in der populären Musik}

Stimme in Gestalt des rhythmisch akzentuierten Sprechgesangs, des Rap, gehört neben den musikalisch-instrumentalen (DJing), pikturalen (Graffiti) und motorisch-kinetischen (Breakdance) Performativen zum Kern der HipHop-Kultur seit ihren New Yorker Anfängen Mitte der 1970er Jahre. In starkem Kontrast zum stetig anwachsenden Strom soziologisch motivierter Studien und literaturwissenschaftlicher Analysen von Rap-Texten bildet die Stimme, und im Besonderen die Stimme als Klangereignis, gleichwohl einen weitgehend blinden Fleck innerhalb der wissenschaftlichen Diskussion. Entlang der mehr oder weniger gewundenen Pfade der Forschung fehlt es somit an der Erschließung und Kartographie eines zentralen Mediums der HipHopKultur.

Einer der Gründe hierfür ist sicherlich, dass die Kategorie der Musik selbst, sprich des Klangereignisses im Sinne eines »primary text (Moore 2001) populärer Musik selber in Frage steht. ${ }^{1}$ Auch die Musik des HipHop, die im Kontext stark performativer, oral tradierter Kultur steht, kann zumindest mit den traditionell notations- und werkorientierten Verfahren musikwissenschaftlicher Analyse schwerlich angemessen beschrieben werden. Und nur wenig mehr verspricht es, Rap sozusagen abzüglich seiner Klanglichkeit als Schrift zu behandeln, als das also, was vom Rap übrig bleibt, wenn er es aufs Blatt Papier geschafft hat - und darum kein Rap mehr ist. HipHop von der Sprache selbst her zu lesen, löst die Rap-Verse nicht nur aus ihrem

1 Vgl. die entsprechenden Thesen von Peter Wicke (2003: 7). Den Begriff des »primary text « erläutert Moore (2001: 35) kurzerhand mit den Worten »which is, [...] what is heard $\ll$. 
sozialen Kontext, sondern auch aus jenem Medienverbund, der für die HipHop-Kultur so charakteristisch ist. Dies kann darum nicht minder problematisch erscheinen als die Konstruktion einer Musik selbst. Über Sprache und Schrift allein ist der Stimme im HipHop ebenso wenig beizukommen wie über Töne und Notenpapier. Dasselbe gilt für Zugänge, welche die Tonträgermusik des HipHop über ihre leitenden Techniken des Cutting, Mixing, Scratching und Sampling analysieren; schließlich bildet das akustische Stimm-Instrument des Rappers innerhalb der Montageästhetik des found sound eine wichtige medientechnologische Ausnahme. Die Stimme des Rappers stellt das Problem Sound - als das entscheidende Problem der Analyse populärer Musik - in anderer Weise als Musik auf der Basis phonographisch gespeicherten Klangs. Wie aber lässt sich über die Stimme sprechen, wenn sie weder rein medientechnologisch noch als bloßes Klangereignis oder gar als Medium von Sprache hinreichend zu erfassen ist?

Einen möglichen Ausweg weist Simon Frith (1996: 183-202) mit seinem Modell der Pop-Voice, welches das Sound-Problem weder einseitig strukturästhetisch noch medientechnologisch auffächert, sondern rezeptionsästhetisch, also im Spannungsfeld zwischen Klangproduktion, Klangcharakteristik und Klangwahrnehmung. Stimme kann, so Frith, unter verschiedensten Vorzeichen gehört werden: als Repräsentant eines Autors oder einer Rollenfigur, als Interpret oder Kommentator, als Ausdrucksmedium, als Verkörperung eines fiktiven Text-Ichs, als Körperlaut oder als Instrument. Vor allem aber hören wir Stimme eben als personal kommunizierendes Medium. Hier schließt Frith an einen breiten Konsens neuerer kulturtheoretischer Ansätze an. Stimme, schreibt etwa die Theaterwissenschaftlerin Doris Kolesch, ist Index des physisch-organischen Zusammenhangs, in dem sie erklingt, ist »eine Spur des Körpers [...]. Dieser Index unterhält einen physischen, materiellen Zusammenhang zum Bezeichneten. Von daher gibt es keine >reine<, körperlose Stimme « (Kolesch 2003: 276). Aber Stimme legt, betont Kolesch, zugleich eine biographische Spur, Niederschlag »auch bestimmter kultureller Praktiken, Normen, Traditionen und Disziplinierungen« (ebd.). Über den Anteil dieser Einschreibungen am persönlichen Stimmklang wird unten noch zu reden sein. Musikwissenschaftlich gesprochen, ist ein Gesangston darum aber in jedem Fall mehr als ein »Ton«, will sagen mehr als ein musikalisches Klangereignis, das sich allein durch seinen Ort innerhalb einer Struktur notierbarer Höhen- und Dauernwerte definiert. „Die reine Stimme ist ein Mythos. Sie ist ohne Verbindung zum Ausdruck und zum Sinn nicht vorstellbar « (Wimmer 1984: 125). Wir hören nicht nur Singen, sondern zumindest in Umrissen immer auch, wer singt, und dies setzt uns in ein je spezifisches Verhältnis zu ihm - obwohl, oder vielmehr weil wir meistens nicht genau 
benennen können, worin dieser Index überhaupt besteht. Denn ist es nicht überhaupt gerade das Nichtqualifizierbare und Atopische der Stimme, was ihre Anziehungskraft hervorruft? So sieht es zumindest der Kulturtheoretiker Mladen Dolar (2004: 218): „Stimme ist mit der größten Bedeutung und ästhetischen Faszination genau an dem Punkt ausgestattet, an dem sie der Strukturierung und Kodifizierung entkommt.«

Insbesondere Sänger der populären Musik haben diese Eigenschaft der Stimme zu nutzen gewusst und produktiv gemacht - mit dem Resultat jenes »ideal of self-expression « (Moore 2001: 182), das oft und gern für wichtige Stile der Pop- und Rockmusik in Anspruch genommen wird. In der klassischromantischen Gesangstradition, darauf weist Frith (1996: 185ff.) sicher zu Recht hin, wird die Spur des empirischen Körpers dagegen wenigstens der Tendenz nach getilgt und, dem Ideal des beseelten Belcanto-Tons folgend, in den Dienst ästhetischer Expression und tönender Form genommen. Es spricht der Komponist oder die Musik, nicht aber die Sängerin als Privatperson mit ihren realen Nöten, Sorgen und Befindlichkeiten. Seit den Kastraten des 17. Jahrhunderts zählt im System Kunstmusik die Darstellung eines bestimmten, durch den Komponisten vorgegebenen Ausdrucks sowie die virtuose Leistung bezüglich Tonhöhe, Geschwindigkeit der Koloraturen und Flexibilität der klangfarblichen Register. Das persönliche Timbre erscheint dagegen als bloße Zutat (und das Gegenbeispiel von Gesangsstars wie Maria Callas trifft insofern nicht den Punkt, als deren Rezeption als quasi öffentliche Privatperson eben wiederum im Modus populärer Kultur stattfindet). In der populären Musik avanciert der Wettstreit zwischen ästhetischer Struktur und Musiker dagegen zum zentralen Merkmal für Qualität und die Stimme darum zuallererst zum Ausweis von Persönlichkeit: »The best pop songs, in short, are those that can be heard as a struggle [...] between the singer and the song « (Frith 1996: 182). Es spricht der Interpret, erfahrbar in den körperlichen Limits ${ }^{2}$ seiner warmen oder näselnden, brüchigen, hauchigen, rauen oder auch gequetscht klingen Stimme, nicht aber oder nachrangig die Komposition und ihre Urheber. Wenn wir »Candy Shop « auflegen, hören wir wohl den Song mit seinen Beats, Samples und Lyrics, erkennen vielleicht sogar den Textautor und früheren Mixtape-Komponisten an Sound und Stilistik. Zunächst aber und vor allem anderen hören wir den Rapper 50 Cent, hören »Jamaica, Queens' realest son«, vordem Crackdealer und Drogenboss, dessen Körper neun Schüsse auffing und überlebte, darunter der Legende nach ein 9-mm-Projektil im Kopf. Und hören, oder vermeinen zu hören, eine Stimme, die uns, was sie auch sagt, genau davon erzählt - Kenner identifi-

2 Zur Bedeutung stimmlicher Limits in der Stimm-Rezeption von Rockmusik vgl. Dai Griffiths Artikel über Bruce Springsteen (Griffiths 2000). 
zieren dazu vielleicht noch die Sound-Designer im Hintergrund, im Fall von »Candy Shop« federführend Scott Storch.

Aber hören wir wirklich Curtis Jackson oder sitzen wir nicht vielmehr etwas ganz anderem auf, nämlich dem musikindustriellen Effekt und marktgenau konstruierten Image 50 Cents? In den auf Authentizität setzenden Stilen populärer Musik, zu denen der HipHop ohne Zweifel gehört, ist das Ideal der Selbstexpression schließlich oft nicht viel mehr als ein angenehmer Mythos - und der Pophörer weiß selber sehr gut, dass er nichts über 50 Cent weiß und wissen kann, was nicht dieser Maschinerie entspringt (vgl. Moore 2001: 188f.). In der Regel allerdings entkommen wir der Dynamik der Stimme trotz allem nicht, bleiben wir der Sehnsucht nach der Übereinstimmung von Kunst und Leben verhaftet. Simon Frith (1996: 183-202) spricht in diesem Zusammenhang vom Hören der Stimme als Person. In seinem Modell der Pop-Voice ergänzt er die »voice as a person« (wir glauben der rappenden Stimme tatsächlich etwas über die reale Person 50 Cent zu entnehmen) durch drei weitere Instanzen oder Möglichkeiten, Stimme zu hören: »voice as a character « (50 Cent rappt seine Verse als Protagonist und Text-Ich mit fiktiven Erfahrungen, oder auch nur als Markenartikel der Musikindustrie, der stimmliche Gesten, Haltungen und ein bestimmtes Timbre im Blick auf kommerzielle Erfolge produziert), »voice as a body« (wir hören die physiologische Basis der produzierten Töne und können ihnen Informationen über den Zustand von Kehlkopf, Resonanzkorpus etc. entnehmen) und »voice as an instrument «. Diese vierte Instanz meint die Stimme als ein je nach persönlichem Vermögen und Gesangstechnik beherrschtes Werkzeug, dem seinerseits ein bei aller Wandlungsfähigkeit begrenztes Klangfarbenspektrum und ein endlicher Tonumfang gegeben ist. Gegenüber der »voice as a person« erscheinen die anderen Stimminstanzen letztlich aber nachgeordnet. Im Zentrum der Wahrnehmung von Stimme in der populären Musik steht für Frith der »struggle between the singer and the song « (ebd.: 182), öffnet sich der Bruch zwischen inszenierter und wirklicher Bedeutung und die Kluft zwischen Star und Mensch.

Für Friths Modell spricht, dass es die umstandslose Ineinssetzung von Körperlichkeit und innerem Selbst ausschließt. Wenn wir die »voice as a body« hören, hören wir auf einer anderen Ebene als der des »inner self « (Danielsen 1997: 279). Auf der anderen Seite verhindert seine scharfe Systematik allerdings die Auseinandersetzung mit den Interdependenzen zwischen den vier Instanzen. Ich will darum an dieser Stelle ein kulturwissenschaftliches Konzept ins Spiel bringen, das »voice as a person« und »voice as a body « weder vorschnell ineins setzt noch trennt, sondern vielmehr als korrelierende Möglichkeiten der Wahrnehmung von Stimme 
verhandelt, die gemeinsam eine entscheidende Funktion der Stimme generieren: die Koordination von Nah-Interaktion. Was also und wie hören wir, wenn wir in der Stimme und durch sie hindurch eine Person vernehmen oder zu vernehmen meinen?

\section{Die Stimmen der Stimme}

In der Topologie von Sprache, Körper und Musik besetzt die Stimme, so drückt es Mladen Dolar aus, den Ort einer allen gemeinsamen Schnittstelle. Stimme ist weder (nur) das eine noch das andere, weder nur Klang noch nur Wort, noch rein physikalische Schwingung. Sie enthält immer etwas mehr, ein zusätzliches Element. Diesen »Überschuss an Wirkung « gibt es »bereits bei dem Körper, der eine Stimme erzeugt « (Dolar 2004: 219). Stimme ist mehr als Sprache, weil sie die Sprache im Stimmklang versinnlicht und mit emotional-affektiven Konnotationen auflädt. Und sie ist zugleich mehr als Körper oder Materialität, da sie diese in kommunizierende Sprachlaute verwandelt. »Die Stimme stammt vom Körper, zu dem sie aber nicht gehört, sie trägt die Sprache, zu der sie aber auch nicht gehört, und doch ist sie in dieser paradoxen Topologie der einzige Punkt, den beide gemein haben « (ebd.: 214). Im Fall der Singstimme, können wir Dolar hier ergänzen, ist dieser Punkt dabei zugleich einer der Musik, womit Gesang eben immer auch mehr als Musik ist. Nicht zuletzt diese besondere, interdisziplinäre Analysemethoden herausfordernde Topologie könnte die Aufwertung der Stimme innerhalb der Kulturwissenschaften erklären, die sich in der letzten Zeit in einer Reihe von Publikationen niedergeschlagen hat (vgl. auch die Anthologien von Jäger-Epping/Linz 2003, Kittler/Weigel/Macho 2002 und Felderer 2003). Medientheoretisch und im Blick auf die populäre Stimme besonders interessant scheint mir in diesem Zusammenhang der bereits erwähnte Aufsatz von Doris Kolesch.

Ausgangspunkt für Kolesch sind einige Beobachtungen zum Mitschnitt einer Lesung von Thomas Bernhardts Der Stimmenimitator durch die 2002 verstorbene Berliner Schauspielerin Marianne Hoppe. Stimme - gemeint ist bei Kolesch immer die Sprechstimme - muss zunächst produktionsästhetisch immer als irreduzibles Klanggemisch betrachtet werden, ja geradezu als Heterophonie, in der sich Stimmen und Stimmsubjekte, Stimmmuster und Sprechweisen überlagern. Autorschaft existiert im Fall der Stimme, wenn überhaupt, nur als plurale. Rezeptionsästhetisch lassen sich dabei, was Koleschs Text nahe legt, ohne selber eine systematische Trennung einzuführen, aber drei Ebenen unterscheiden, auf denen Stimme hauptsächlich 
gehört wird. Zugunsten einer übersichtlichen Darstellung spreche ich im Folgenden von der Stimme als Medium von Sprache, der Stimme als Körper und der biographischen Stimme. Kolesch thematisiert zunächst die ersten beiden: »Wir hören nicht nur, was Marianne Hoppe sagt, wir hören Marianne Hoppe atmen, hören das Schmatzen und die Bewegungen ihrer Lippen, ihrer Zunge und ihres Gaumens, hören das leibliche Fundament des Sprechens und der Sprache (Kolesch 2003: 269).

Neben der kommunizierten Sprache sind es also Zunge, Gaumen, die Klangproduktion des Stimmapparats mitsamt seinen Resonanzräumen in Kehlkopf, Hals, Kopf und Rumpf, die einer Stimme Kontur geben. Innerhalb ihrer physisch-akustischen Möglichkeiten generieren sie das Klangmaterial für das Phonem, den Sprachlaut. Noch ganz im Einklang mit Frith - dessen Modell sie aber nicht anspricht - ist es somit auch für Kolesch nicht zuletzt der Körper, den wir in der Stimme hören und der sie ausmacht und sschreibt<. Die Stimme als Medium von Sprache wird erzeugt und zugleich in ihren klanglichen Eigenschaften markiert und ergänzt durch die Stimme als Körper. Allerdings, und damit insistiert Kolesch auf einer differenzierteren Systematik als Frith, ist die Stimme selber keineswegs statisch und somit eben alles andere als ein nach Belieben zu spielendes, je nach Übungsleistung beherrschbares Instrument mit einem bestimmten Korpus, der Umfang, Register und Klangfarben physikalisch determiniert. Darauf verweist die dritte Ebene, auf der wir Stimme hören. Jede Stimme nämlich erklingt immer auch als ein Echo des Stroms biographischer Kontinuitäten und Zäsuren, kultureller Praktiken und Traditionen sowie des Alters der Sprechenden, ihres Geschlechts, ihrer Krankheiten, mentalen Stimmungen, Befindlichkeiten, ihres Temperaments, nicht zu vergessen schließlich ihres Berufs - in der Stimme von Marianne Hoppe unüberhörbar präsent in der professionellen Sprechtechnik. Das Leben schreibt den Körper, der die Stimme schreibt; das Instrument Stimme - und nicht nur die Fähigkeit, es zu bedienen unterliegt selber stetigen, biographisch und das heißt auch: kulturell und sozial bedingten Veränderungen. Denn anders als bei allen anderen Instrumenten bedient und spielt der Körper nicht nur, er wird zugleich gespielt. Es ist die biographische Erfahrung, die in ihm immer wieder Resonanzen weckt oder auch abdunkelt und verschließt, und dies zu jeder Zeit neu und anders. Durch sie hören wir nicht den oder irgendeinen Körper, sondern eben »einen bestimmten Körper, dem wir ein Geschlecht, ein Alter, einen sozialen Status, bisweilen auch eine regionale Herkunft zuschreiben«, und wir hören »ein gelebtes Leben, die Spuren eines Jahrhunderts, die in der Reibung, der Verbrauchtheit und der gelassenen Souveränität dieser Stimme nachklingen«. »Zu hören gibt die Stimme Hoppes darüber hinaus eine 
bestimmte Technik und Tradition, eine geschulte und geübte Praxis, ja Virtuosität des schauspielerischen Sprechens« (alle ebd.: 269).

Dieser Verkettung von biographischer und körperlicher Stimme fügt Friths Modell eine so interessante wie entscheidende Pointe hinzu: Weder die Sprache noch die Kultur noch der Körper können für sich als Letztinstanzen des Sprechens und des Mediums Stimme in Anspruch genommen werden. Die Stimme bringt zwar die »Spur des Körpers in der Sprache (ebd.: 267) zum Klingen. Wir erkennen eine bestimmte Person wesentlich schneller und sicherer an ihrer physiologischen Signatur als an ihren Aussagen und Meinungen. ${ }^{3}$ In dieser Spur selber aber sind kulturelle Praxis und organische Materialität immer koexistent. Die personale Signatur der Stimme erscheint als ein komplexes Zusammenspiel von Körper und Kultur, von sozial kodiertem und physiologisch bedingtem Klang und stellt dadurch die Unterscheidung zwischen »body « und »person«, in Friths Modell noch stabile Differenzmerkmale, grundlegend in Frage.

Eine klare Grenze lässt sich darum im Blick auf die Stimme nur in medientheoretischer Sicht ziehen. Sie verläuft zwischen Sprachlaut und Stimmlaut und schreibt der Stimme zwei wesentliche mediale Funktionen zu: Stimme ist einerseits Medium von Sprache und zugleich ein zweites, komplexes Medium, in dem biographische Stimme und Körper-Stimme zusammenfließen. Diese zweite, personale Stimme ist an der Kommunikation über sprachliche Zeichen unbeteiligt und markiert stattdessen die individuelle Signatur der Stimme, ihren persönlichen Sound - eine Unterscheidung, mit der Kolesch an die Tradition des funktionalen Strukturalismus anknüpft, die auch Dolar aufgreift, wenn er zwischen dem sinnhaften Sprachlaut und einem »Laut-Überschuss« trennt, »der keinen Sinn ergibt, der einfach so da ist, spaßeshalber, um seiner Schönheit willen, zum reinen Vergnügen« (Dolar 2004: 200). Simon Frith spricht seinerseits in einem früheren Text von »noises around the words« (Frith 1987: 35). Allerdings muss dieser überschüssige Laut selber eben als immer schon mit Kultur und Sprache vermittelt gedacht werden - das zumindest ist die Präzisierung, welche die Definition der personalen Stimme als Lautebene nahe legt, in der sich Stimm-Körper und Stimm-Kultur gleichermaßen einschreiben, bedingen und formen. Und die zweite, nichtsprachliche Ebene der Stimme kann deswegen gerade nicht als bloße Stockung oder Friktion zwischen der Materialität des Körpers (Genogesang) und den kulturellen Kodierungen (Phänogesang) beschrieben werden, wie es Barthes berühmte Figur des »grain de la voix« versucht. Barthes entwickelt in seiner antimetaphysischen Wende zur Materia-

3 Darum auch die erkennungsdienstliche Nutzbarkeit der Stimme als akustischer ,Fingerabdruck<. 
lität der Stimme letztlich die neue Metaphysik eines kontextfreien stimmlichen Klangs selbst, verbunden mit der Vorstellung einer frei von kulturellen Disziplinierungen erklingenden Stimme-in-der-Stimme, dem »Genogesang « (vgl. Barthes 1990). Demgegenüber verweisen die kulturellen Spuren, Einflüsse und Markierungen, wie sie in jeder Singstimme mitschwingen und hörbar sind, auf die Untrennbarkeit stimmlicher Physis von den Niederschlägen biographischer Erfahrung.

Im Blick auf die Stimme als Medium von Persönlichkeit bedeutet das eine entscheidende Weichenstellung. Denn der persönliche Stimmklang lässt sich auf dieser Grundlage nicht anders als ein pluraler Sound definieren, in den sich Organisches und Soziales, Materie, Körper und Geschlecht, Alter, Herkunft und nicht zuletzt auch Ethnisches einschreiben. Er erscheint somit als das Resultat immer auch kollektiver Prägung. Noch im sublimsten Kunstgesang der großen »black opera diva« Marian Anderson bleibt eine letzte Spur ihrer Gospel-Sozialisation hörbar, wie die Blues-Vokalisation in der Stimme von Thomas A. Dorsey, die den modernen Gospel für immer veränderte und popularisierte. Es scheint mir darum nahe liegend, Koleschs Beobachtungen zur Sprechstimme im Blick auf die singende Stimme zu ergänzen. Ihr zufolge erscheint der Lautüberschuss des Stimmklangs »im Wie des Sagens: in seinem Rhythmus, seiner Musikalität, seiner Klangfarbe und Modulation « (Kolesch 2003: 279). Nimmt man diese klanglichen Parameter ernst, definieren sie die Signatur der personalen Singstimme als Spiegel und Spielfeld nicht zuletzt musikstilistischer Einflüsse und Distinktionen. Auch die Persönlichkeit der Singstimme erscheint nicht im Was des Singens, sondern im Wie. Aber sie artikuliert sich trotzdem und notwendig innerhalb eines ästhetisch-musikalischen Zeichenkontexts als Widerklang und Umschrift existierender Ausdrucksmodelle.

Moores Kritik am »ideal of self-expression« (2001: 182) des Rock ist darum nur zu bekräftigen. Wenn die klingende Stimme, ganz im Sinne von McLuhans berühmter Denkfigur, mit der personalen Stimme ein Medium hervorbringt, das in seinen hörbaren Reibungen und seiner Elastizität, seinem Knacken und Brechen, aber auch den kulturellen Prägungen, die in ihm hörbar werden, zunächst einmal vor allem den eigenen materialen Zustand offen legt, dann ist seine Botschaft nichts anderes als eben das Medium Stimme selber - welches der Zuhörer anschließend als Echo erlebten Lebens und vergangener Einschreibungen auffassen kann. Die personale Stimme ist, so betrachtet, sogar das genaue Gegenteil eines Mediums bewusster Selbstexpression. Denn über den Klang der personalen Signatur entscheiden die mehr oder weniger kontingenten Wechselfälle einer gesamten (Stimm-)Biographie, entscheiden Herkunft, musikalisches Umfeld, 
organische Konstitution usw. Aktuelle Absichten des kommunizierenden Subjekts haben darauf keinen Einfluss. Mit Bernhardtscher Konsequenz gesprochen, ist der Sender - als Instanz der Intentionen und des Ausdruckswillen - in der personalen Stimme sogar vollständig abwesend. Den persönlichen Klang seiner Stimme zu modifizieren endet noch immer in der Verstellung; nicht zuletzt darauf verweist Bernhardts Erzählung vom Stimmenimitator, der alles nachzuahmen versteht, nur seine eigene Stimme nicht.

Erst in diesem Sinne, und nicht in dem eines authentisch angelegten Ausdrucks oder eines bloßen Körper-Signals, wird die Stimme als Authentizitätssignatur hörbar. Was aber bedeutet das hinsichtlich ihrer medialen Funktion? Auch zu dieser Frage macht Kolesch einen Vorschlag. Im Anschluss an Roland Barthes' Versuch, Hören und Berühren, Sprechen und Kuss als miteinander verschränkte Modi der Intersubjektivität zusammen zu denken, begreift sie das Hören der Stimme als Angebot zu Nah-Interaktion und Identifikation: Stimme-Hören ist »nicht bloß Kommunikation unter Menschen, sondern es ist eine spezifische, jeweils unterschiedlich zu bestimmende Form der Begegnung, der Nähe (oder auch der verweigerten, gefürchteten Nähe« (Kolesch 2003: 279). Das Hören der personalen Stimme ist demnach kein nur passiv-rezeptiver Vorgang, wie er herkömmlichen Hörmodellen zugrunde liegt, sondern ein affektiver Appell mit dem Potential, Subjekt und Objekt, Sänger und Hörer zu involvieren. Oder, in den Worten von Roland Barthes: »Die Aufforderung zum Zuhören ist das vollständige Ansprechen eines Subjekts: Sie stellt den gleichsam körperlichen Kontakt zwischen diesen zwei Subjekten (durch die Stimme und das Ohr) über alles: Sie schafft die Übertragung: >Hör mir zu< heißt: Berühre mich, wisse, dass ich existiere (Barthes 1990: 255; Hervorhebungen im Original).

Ich meine, dass mit dieser Überlegung eine grundlegende Funktion der personalen Stimme auch in der populären Musik beschreibbar wird. Ob ich aus der Stimme herausspüre, wie anders die Sängerin doch ist, als ihr knalliges Image nahe legt, oder ob sie für meine Ohren ganz so singt, wie ich sie mir als Person vorstelle - ob es also zum »struggle [...] between the singer and the song (Frith 1996: 182) kommt oder zur romantischen Bestätigung der Einheit von Kunst und Leben - in beiden Fällen gerate ich in ein spezifisches Nähe-Erlebnis mit einem Subjekt, das sich mir in seiner personalen Stimme unverhüllt präsentiert als authentisch empfindendes Subjekt und Mensch hinter dem artistischen Vorhang. Die Stimme übernimmt dabei eine Funktion, die im Sinne der Psychoanalyse Jacques Lacans, auf die sich auch Barthes Anthropologie des Sprechens und Küssens bezieht, als Vorgang befreienden Genießens begriffen werden kann. In diesem intensiven Genuss streift das Subjekt seine als Defizit erlebten Begrenzungen ab, 
lässt seine Kontingenzerfahrungen hinter sich und öffnet sich in der Begegnung mit einem Objekt, mit dem es dabei tendenziell verschmilzt. Nach Lacan ist die Stimme dabei eines der Primärobjekte, die das Genießen ermöglichen, weil sie das Subjekt zugleich regulieren und nicht in den Selbstverlust treiben. Populäre Musik erlaubt, weil sie den persönlichen Klang der Stimme und damit den Zugang zur Nah-Interaktion mit dem Sänger akzentuiert, solche Situationen des Genießens in besonderer Weise. In ihrem Gesang berührt mich die Sängerin und liebkost mich mit ihrer Stimme, gibt sich mir zu erkennen, und das ganz buchstäblich. Ihr Körper expandiert in die vibrierenden Schwingungen der Luft und gerät so in Kontakt mit meinem. Umgekehrt kann es gelingen, die singende Stimme nicht mehr objekthaft zu hören, sondern vollständig mit ihr zu verschmelzen und den Star damit zum Medium eigener Expressions- und Emotionslust zu machen. Indem ich der personalen Stimme glaube und mich ihr überantworte, repräsentiert sie zugleich meine eigene.

Die Identifikation mit dem Star stellt, darüber dürfte Konsens herrschen, eines der gängigsten Rezeptionsmuster populärer Musik dar. Dass dabei aber nicht zuletzt die musikalischen wie kulturellen Markierungen der personalen Stimme eine Rolle spielen, will ich nun im Blick auf 50 Cent als dem Repräsentanten eines neuartigen Phänotyps der Rap-Stimme im Mainstream-HipHop der 2000er Jahre zeigen. Die personale Stimme dieses Rappers hält in ihrem auf bestimmte soziale und ethnische Kontexte verweisenden Zuschnitt präzise Identifikationsangebote bereit, mit denen sie breite Hörerschichten binden, auf ihre historischen Wurzeln verweisen und diese zugleich kommentieren kann.

\section{Stimme im HipHop}

Die Musik des HipHop lässt sich aufgrund ihrer Rekombinationsästhetik einem Typus hybrider Musik zuordnen, den Quincy Jones einmal »renegadetype music « (Jones 1990: 167) genannt hat. Trotz dieses Wiedergängertums macht der HipHop Persönlichkeit und Authentizität zum Maßstab für die Akzeptanz seiner Akteure, verstanden allerdings als performative, also immer wieder neu zu erkämpfende und auszuhandelnde Attribute. Dass dies in der Battle um den virtuosesten Vortrag von Persönlichkeit ritualisiert ist, macht das postmoderne Element des HipHop aus und unterscheidet ihn von anderen, auf statische Authentizität und Star-Aura setzenden Stilen. Noch das notorische Dissing führender HipHop-Musiker untereinander kann als Ausspielen dieser Wettbewerbsstruktur auf der Basis global zirkulierender 
Medien begriffen werden. Ich will den strapazierten Authentizitätsdiskurs an dieser Stelle aber nur insofern aufgreifen, als er die Bedeutung der Stimme in der HipHop-Musik beleuchten kann.

Die Rap-Stimme wird in dem Maße zum zentralen Aufmerksamkeitsmedium des HipHop, in dem die Rapper in den späten 1970er Jahren die DJs, Sprayer und Breakdancer an Popularität zu übertreffen beginnen. Zumindest für einige Zeit erscheint es möglich, über ethnische, soziale und räumliche Grenzen hinweg einer glaubwürdigen öffentlichen Stimme der afrikanisch-amerikanischen Unterschicht Gehör zu verschaffen. Diesen Status haben Stimme und Rap bekanntlich nicht von Anfang an. Wie David Toop (1994: 85-120) berichtet, beschränkte sich der Rap anfänglich auf die Funktion, für Stimmung zu sorgen und in der Tradition des jamaikanischen Toasting zum Tanz über die kunstvoll ineinander gemischten Breakbeats zu animieren. Textinhalte waren demgegenüber eher gleichgültig. Für den Augenblick zusammengeschmiedet und wesentlich mehr mit Rhythmus und Stimmklang beschäftigt als mit Botschaften, bestand der Rap im Wesentlichen aus soundorientierter Spontanlyrik. Was zählte, war die Erfindung möglichst ausgefallener Reime in Echtzeit, in Verbindung mit zunehmend virtuosen stimmlichen Skills bis hin zur Human Beat Box eines Doug E. Fresh. Erst durch die zunehmende Virtuosität der DJ-Techniken, die immer mehr Aufmerksamkeit und Konzentration beanspruchten, kam es zu einer richtungsweisenden Aufgabenteilung - und zur Geburtsstunde des MC, der die gerappten Texte ausdehnte und schließlich mehr und mehr in den Mittelpunkt rückte.

Dieser Übergang von einem MC-Typus, dem allein der Flow der Reime seinen Status innerhalb der lokalen Teilkultur sichert, zu einem neuen, der sich durch die stimmliche Artikulation ethnisch und soziokulturell markierter Persönlichkeit auszeichnet, ist mit dem durchschlagenden Erfolg von Grandmaster Flashs »The Message« (1982) abgeschlossen. Die schwierige Situation afrikanisch-amerikanischer Jugendlicher in den Großstadt-Ghettos, »close to the edge «, ist hier sprachlich unübertrefflich thematisiert und in eine schlagende Textbotschaft gegossen. Die eigentliche politische Wirkung des Rap ist jedoch schon deutlich früher anzusetzen.

Ich möchte vorschlagen, sie nicht zuletzt als eine Mikropolitik der Stimme zu verstehen. In dieser Perspektive erscheint die personale Stimme als dasjenige Medium, welches das neue kulturelle Selbstbewusstsein der jungen HipHopper artikuliert, und die Botschaft des frühen Rap als eine Botschaft nicht der Sprache, sondern der Stimme. Obwohl, oder besser, gerade weil der frühe, in seinen Texten noch ganz unpolitische Rap Leben und Körper, Ghetto-Biographie und ethnische Differenz hörbar macht, ohne sie 
dabei in politische Rhetoriken wie die der Bürgerrechtsbewegung zu spannen, erhält er das Gewicht einer mikropolitischen Demonstration, die ihre Brisanz eben durch das Zusammenspiel von biographischer Stimme (hier die Stimme des Ghettos) und Stimme als Körper (nämlich als afrikanisch-amerikanischer Körper) entfaltet. Entscheidend dabei ist, dass es eben keine bloßen Spiegelungen eines »inner self « sind, sondern kollektive Einschreibungen und Markierungen, welche die persönlichen Stimmen der jungen Rapper zu Repräsentanten ihrer Kultur aufwerten. Erst als auditives Gedächtnisfeld der "young black urban culture « bringen die Rap-Stimmen ein Ausdrucksmodell hervor, das über den konkreten historischen und geographischen Kontext hinweg zur Nah-Interaktion einlädt.

Die Erfolgsgeschichte dieses Modells dauert bis heute an - unabweisbar und unüberhörbar jedenfalls, dass die ethnisch markierte Ghetto-Stimme als Schnittmuster nahezu aller späteren, politisch agierenden HipHop-Stile gelten muss. Auch 50 Cent bietet ein gutes Beispiel dafür, sofern sich seine Stimme nicht zuletzt als latenter Rekurs auf die HipHop-Anfänge artikuliert. Angesprochen ist mit ihm allerdings eine Konstellation von stimmlichem Verweis und Kommentar, die zugleich ein neues, bislang kommerziell ungemein erfolgreiches Schnittmuster der Rap-Stimme ins Spiel bringt. Was lässt sich über 50 Cents Stimme sagen? Ins Ohr geht zunächst vor allem die angesprochene Diskrepanz zwischen seinem sorgfältig präparierten Image als misogyner Hardcore-Rapper und einer defensiven, geradezu anti-militanten Stimmästhetik, mit der sich 50 Cent weit vom bekannten Stereotyp des Gangsta oder Hustler entfernt. Dabei kann 50 Cent eigentlich mit einer Street- und Ghetto-Biographie aufwarten, die ihn zum Hardliner prädestinieren sollte. "Ich landete auf den Straßen, als ich eigentlich noch ein Baby war «, heißt es auf seiner deutschen Homepage (www.50cent.de; Zugriff: 14.12.2005). »Ich war erst zwölf. Die Jungs aus meiner Nachbarschaft passten auf mich auf, und ich kam schon früh mit schrägen Geschäften, schrägen Leuten in Kontakt« (ebd.). Und natürlich beherrscht 50 Cent auch das mit Gewaltandrohungen verknüpfte Dissing: »Ich will, dass sich meine Konkurrenten verziehen. [...] Ich will, dass mir verdammt noch mal alle Rapper aus dem Weg gehen« (ebd.). Den Weg aus dem Slum führen ihn demnach DJs von Rang und Ehren wie Jam Master Jay von Run DMC und vor allem Eminem, der ihm nach dem um ein Haar tödlichen Attentat eine neue Chance gibt: "Letztlich hat Eminem mich gerettet, als er mich von der Straße holte. Wenn man nämlich lange genug auf der Straße sitzt, dann muss eines Tages etwas passieren: Entweder man bringt einen um, wird gekillt, oder landet im Gefängnis. Die Musik war mein Weg aus diesem Teufelskreis« (ebd.). Seine geradezu softe, jungenhafte Stimme, die er in 
"Candy Shop«, aber auch in schnelleren und in Tempo, Beatstruktur und Sound härteren Songs produziert, will diesem Hintergrund allerdings so gar nicht entsprechen. Ich möchte abschließend zeigen, dass 50 Cent dadurch ein prominentes Ausdrucksmodell der HipHop-Geschichte wenn nicht vollständig umschreibt, so doch entscheidend verschiebt.

\section{Stimme in $\gg$ Candy Shop}

Text, Bild und Musik des Songs sind entlang des zugehörigen Videoclips schnell beschrieben: die topologische Selbstinszenierung des Stars als sexuell potenter "african-american hero«, von süßen Nymphen umschwärmt: »Yeah... / Uh huh / So seductive«. Die luxuriöse Villa als Schauplatz nächtlicher erotischer Szenen und obszöner Doppeldeutigkeiten: »Got the magic stick, I'm the love doctor [...] / I melt in your mouth girl, not in your hands (haha)«. Fließende Zuckerströme, geheimnisvolle Verwandlungen und Schnitte, die den Rapper immer wieder aus den Armen der jungen Frauen samt ihrer wunderbaren Sirupwelt reißen. Das Pendeln zwischen Akteursund Voyeurs-Perspektive: "Lights on or lights off, she likes it from behind / So seductive, you should see the way she wind / Her hips in slow-mo on the floor when we grind «. Und das prosaische Ende, als plötzliches Erwachen in eine reichlich öde Fast-Food-Realität inszeniert. Im Kontrast zu der luxuriösen Ausstattung des Bildraums zeigt sich die auditive Seite dabei ganz von einer Reduktions-Ästhetik bestimmt. Die Musik beschränkt sich auf die Kombination weniger kurzer Samples und verzichtet auf jede umfänglichere Klangschichtung. Ein eintaktiger, schwerer Bass-Loop ist zu hören, Handclaps sowie eine Klangfläche mit zwei modalen Akkorden, kurzen, melodischen Orientalismen und regelmäßig eingestreuten Shouts, mehr nicht. Für die tonale und rhythmische Struktur der Stimme gilt ähnliches; die RapVerse kommen ohne jeden Anflug energetisch treibender, gegen Takt- oder Beatakzente opponierender Rhythmen aus. Virtuose stimmliche Skills sind in "Candy Shop « nicht mehr im Angebot. Und nur der siebentönige Refrain mit seiner einfachen Dreiklangsstruktur wird innerhalb des Lustpalasts gesungen, während die gerappten Verse selber als Kommentar aus der dunklen

(und kalten?), jedenfalls distanzierten Nacht erklingen und dies in einem eher wattigen, aus dem Zentrum geholten und wenig komprimierten Sound.

Und doch lässt mich die rappende und singende Stimme in »Candy Shop« aufhorchen, wenn auch aus einem anderen Grund. Was also höre ich? Wenn ich mich auf ihren personalen Klang einlasse, höre ich die Stimme von 50 Cent einerseits als stark jugendlich-männlich und körperlich konnotierte 
Stimme, die genügend Merkmale des Street-Talk aufweist, um sich deutlich in die Ghetto-Tradition der Rap-Stimme zu stellen. Andererseits aber scheint mir ihre weiche und gleichzeitig tiefenorientierte Klangfarbe, ihre Entspanntheit, vernuschelte Aussprache, nachlässige Tongebung und die nur halb ausgespielte Lautstärke ein stimmliches Ausdrucksmodell zu verabschieden, das lange unbestritten im Zentrum der HipHop-Geschichte und in Teilen der afrikanisch-amerikanischen Popgeschichte überhaupt stand. Noch die Stimmen der lässigsten, mit ausgewiesenen Statussymbolen behängten Gangsta-Rapper der 1990er und frühen 2000er Jahre klingen unverhüllt nach Gefährdung, Legitimitätsdruck, auftrumpfender Selbstbehauptung und aggressiven Statuskämpfen. 50 Cents Stimme entfernt sich in meinen Ohren so weitgehend von diesem Modell wie kaum ein anderer Rapper vor ihm. An die Stelle der kämpferischen Aufstiegs- und Erfolgsbehauptung tritt bei ihm stattdessen die genussvolle Affirmation umfassenden, dabei ganz traditionell erotisch und ökonomisch kodierten Erfolgs.

In dieser Perspektive höre ich »Candy Shop « als eine lustvolle, obgleich nicht ohne dunkle Untertöne artikulierte Demonstration des Gelingens, mit der 50 Cents Stimme die Realität seines definitiven Entkommens aus dem Ghetto-Dasein feiert und ausstellt. „Candy Shop « ist nicht mehr das Versprechen oder die Behauptung gesellschaftlichen Aufstiegs, sondern seine genießerische Zelebrierung (und, als potentieller Verkaufsschlager, Garant für seine Dauer). Trotz der üppigen Bilder ist es dabei eben die personale Stimmsignatur des Rappers und Sängers Curtis Jackson, die hier das Wort führt. Sie legt die entscheidende, kulturell wie biographisch konnotierte Spur. Und sie produziert damit nebenbei ein Identifikationsangebot für eine Hörerschaft, die keineswegs schwarz, sozial unterprivilegiert und afrikanisch-amerikanisch sein muss. Die Affirmation des Genießens lässt sich in vielen Kontexten feiern - der Möglichkeit, sich mit der Stimme 50 Cents zu identifizieren, ist damit weit die Tür geöffnet.

Ich finde es verlockend, die Titelmetapher in diesem Sinne als selbstreflexive Pointe des Songs aufzufassen. Denn »Candy Shop« - wie auch 50 Cent - ist natürlich selber nichts anderes als ein höchst erfolgreich verführendes Produkt und Musik-Konfekt. HipHop im Candy Shop - gerade der Clip inszeniert Reichtum und sexuelle Verfügungssouveränität allerdings nicht ohne Brechungen. Die Sex'n'Sugar-Welt, in der zugleich mehr Verführung steckt als Erfüllung, ist, so zeigt sich am Schluss, nur ein vergänglicher Traum, aus dem der Protagonist schließlich recht unsanft erwacht. Trostreich, dass die düstere Wirklichkeit der Drive-Ins und ihrer missgelaunten Angestellten dann immerhin ein Versprechen enthält. Wie sich in den letzten Sekunden des Spots zeigt, landet der Protagonist in einer zwar prosa- 
ischeren, aber doch finanziell noch immer potenten Gegenwart. Das sonore Aufheulen des dunklen, lackglänzenden Sportwagens, mit dem er aus dem letzten Bild fährt, verheißt eine süße Zukunft gediegenen ökonomischen Erfolgs, als die Verlängerung des Traums mit anderen Mitteln. Die Nachzeit aller Aufregungen, das scheint mir hier angedeutet, muss man sich eben auch leisten können. So: Get Rich Or Die Tryin'...

\section{Literatur}

Barthes, Roland (1990). Der entgegenkommende und der stumpfe Sinn. Kritische Essays III. Frankfurt/M.: Suhrkamp.

Danielsen, Anne (1997). »His Name was Prince: A Study of Diamonds and Pearls.« In: Popular Music 16, H. 3, S. 275-291.

Dolar, Mladen (2004). "Sechs Lektionen über Stimme und Bedeutung. "In: Phonorama. Eine Kulturgeschichte der Stimme als Medium. Hg. v. Brigitte Felderer. Berlin: Matthes \& Seitz, S. 199-222.

Epping-Jäger, Cornelia / Linz, Erika (Hg.) (2003). Medien/Stimmen. Köln: Dumont.

Felderer, Brigitte (Hg.) (2004). Phonorama. Eine Kulturgeschichte der Stimme als Medium. Berlin: Matthes \& Seitz.

Frith, Simon (1996). Performing Rites: On the Value of Popular Music. Oxford: Oxford University Press.

Frith, Simon (1987). »Why do Songs have Words? « In: Lost in Music: Culture, Style and the Musical Event. Hg. v. Avron White (= Sociological Review Monograph 34). London: Routledge, S. 77-106.

Griffiths, Dai (2000). "Three Tributaries to >The River «. In: Reading Pop. Approaches to Textual Analysis in Popular Music. Hg. v. Richard Middleton. Oxford: University Press, S. 192-201.

Jones, Quincy (1990). Listen up: The Many Lives of Quincy Jones. New York: Warner Books.

Kittler, Friedrich / Weigel, Sigrid / Macho, Thomas (Hg.) (2002). Zwischen Rauschen und Offenbarung. Zur Kultur- und Mediengeschichte der Stimme. Berlin: Akademie-Verlag.

Kolesch, Doris (2003). »Die Spur der Stimme. Überlegungen zu einer performativen Ästhetik.«In: Medien/Stimmen. Hg. v. Cornelia Epping-Jäger und Erika Linz. Köln: Dumont, S. 267-281.

Moore, Allan F. (2001). Rock. The Primary Text: Developing a Musicology of Rock. Aldershot: Ashgate (2. Aufl.).

Poschardt, Ulf (1997). DJ-Culture. Diskjockeys und Popkultur. Reinbek bei Hamburg: Rowohlt.

Toop, David (1994). Rap Attack. African Jive bis Global HipHop. München: Heyne.

Wicke, Peter (2003). »Popmusik in der Analyse.« In: Acta musicologica 75, H. 1, S. 107-126.

Wimmer, Michael (1984). "Verstimmte Ohren und unerhörte Stimmen.«In: Das Schwinden der Sinne. Hg. v. Dietmar Kamper und Christoph Wulf. Frankfurt/M.: Suhrkamp, S. 118-134. 


\title{
Diskographie
}

50 Cent (2005): »Candy Shop«, Shady/Aftermath/G-Unit, LC06404.

\begin{abstract}
In contrast to the voice in classical music, which is supposed to be a medium of the composer or the artwork, the voice in popular music is often described as an expression of the singer's »inner self«. According to Roland Barthes' idea of the corporal dimension of the voice, this "personal " voice is often associated with the body. In this article a different model is developed, following concepts by Doris Kolesch and Simon Frith, who describe the personal voice as a mixture of the physiological and the biographical voice: life shapes the body, which shapes the voice. Absent in this concept of the personal voice are language and music as symbolic systems of expression. The singing voice is always working with models of expression, which are connected with musical styles and forms. This phenomenon is explained by the example of the micropolitics of the voice in early hip-hop and 50 Cent's song »Candy Shop«.
\end{abstract}

